\title{
Comparison of the effect of polyethylene glycol and simultaneous administration of polyethylene glycol with probiotics in the treatment of chronic functional constipation: a clinical trial
}

\author{
Karamali Kasiri $^{1 * \mathbb{(})}$,Morteza Sedehi $^{(\mathbb{D}}$, Sheida Mortazavi ${ }^{(\mathbb{D}}$ \\ 'Department of Pediatrics, Shahrekord University of Medical Sciences, Shahrekord, Iran. \\ ${ }^{2}$ Department of Statistics, Shahrekord University of Medical Sciences, Shahrekord, Iran. \\ ${ }^{3}$ Student, Shahrekord University of Medical Sciences, Shahrekord, Iran.
}

*Corresponding Author: Karamali Kasiri, Shahrekord University of Medical Sciences, Shahrekord, Iran. Tel: 09138889789 Email: kasiri207@yahoo.com

\begin{abstract}
Background and aims: Constipation is one of the most common issues in pediatrics. The aim of this study was to compare the effect of polyethylene glycol and the simultaneous administration of polyethylene glycol along with probiotics in the treatment of chronic functional constipation.

Materials and Methods: In this randomized double-blind clinical trial, 150 children with chronic functional constipation referred to Imam Ali Clinic affiliated with Shahrekord University of Medical Sciences from 2017 to 2018 were included. The first group (A) consumed $1 \mathrm{~g} /$ $\mathrm{kg}$ of body weight per day of water-soluble polyethylene glycol powder and the second group (B) consumed a probiotic powder and $1 \mathrm{~g} / \mathrm{kg}$ of body weight of the water-soluble polyethylene glycol powder daily. In weeks 0,1 , and 2, questionnaires were filled out by the parents of the children and the data were analyzed.

Results: The results showed that there was no significant difference in any of the variables between groups $\mathrm{A}$ and $\mathrm{B}(P=0.07)$ including the frequency of fecal excretion in week $1(77 \%$ and $71 \%)$ and week $2(4 \%$ and $5.4 \%)$ and stool consistency in week $1(4 \%$ and $6.7 \%)$ and week $2(86.7 \%$ and $92 \%)$. Moreover, there was no significant difference between groups A and B in any of the variables frequency of painful excretion in week 1 ( $74 \%$ and $73 \%$, respectively) and week $2(5.3 \%$ and $4 \%$, respectively), frequency of abdominal pain in week $1(61.3 \%$ and $49.3 \%$, respectively) and week $2(4 \%$ and $5.3 \%$, respectively), and the frequency of fecal incontinence in week $1(22.77 \%$ and $18.7 \%$, respectively) and week $2(6.7 \%$ and $1.3 \%)(P>0.05$, respectively).

Conclusion: Our results indicated that the administration of probiotic supplement (Lactobacillus Rhamnosus, Lactobacillus acidophilus, Lactobacillous Bulgaricus) has no effect on the improvement of symptoms in children with chronic constipation.

Keywords: Probiotics, Functional constipation, Polyethylene glycol
\end{abstract}

Received: 12 November 2019, Accepted: 25 January 2020, ePublished: 29 June 2020

\section{Introduction}

Constipation is a difficulty of stool excretion, painful excretion, or stool retention less than two times in a week (1). Acute constipation refers to painful excretion of stool. The acute constipation may cause closure of the intestine that may require surgery.

It has been reported that nearly 5\%-20\% of individuals suffer from constipation (1). However, the prevalence of functional constipation is different in different societies. In Iran, the prevalence of constipation was reported to range from 1.4 to $37 \%$ and the prevalence of functional constipation ranged from 2.4 to $11.2 \%$ (2). It seems that various factors are involved in the pathogenesis of the disease, including sex, age, socio-economic status, and education level. The prevalence of constipation among children was reported to range from 3-8\% (1). However, this is different in different age groups. A study has reported a prevalence of $16 \%$ for children aged 22 months old, and another study reported that $34 \%$ of children aged 4-11 years old suffered from constipation (3). The prevalence of constipation depends on family history, nutritional habits of individuals, age, sex, and stress (4).

There are various factors for constipation that are categorized into two types, organic and functional. Organic factors include psychological, musculoskeletal, bowel neuromuscular, spinal, pharmaceutical, metabolic, bowel, and connective tissue disorders. As an organic factor, family history accounts for 5\%-28\% of children with constipation and the prevalence of constipation is more common in monozygotic twins than in dizygotic twins. This disease occurs in all social classes and other factors including genetics, socio-economic status, low fiber consumption,

(C) 2020 The Author(s); Published by Shahrekord University of Medical Sciences. This is an open-access article distributed under the terms of the Creative Commons Attribution License (http://creativecommons.org/licenses/by/4.0), which permits unrestricted use, distribution, and reproduction in any medium, provided the original work is properly cited. 
and inadequate liquid consumption are among the various causes of constipation (5-7). Functional factors include cancers, diabetes, thyroid dysfunction, physical inactivity, pharmacotherapy, and pregnancy (1).

The effect of probiotics was also investigated in previous studies. Probiotics are living microorganisms that produce beneficial effects on the gastrointestinal system by balancing bowel microbial flora if administered in adequate amounts (8).

The effect of probiotics on the treatment of functional constipation has been confirmed in recent studies. These studies showed that this effect was due to the reduction of $\mathrm{pH}$ of the colon (9).

Polyethylene glycol is an osmotic laxative that is absorbed in small amounts from the gastrointestinal tract (10). It is the most effective and safest laxative that has long been administered to children (11). Polyethylene glycol is a water-soluble polymer with a high molecular weight that can produce hydrogen chains with 100 molecules of water in each polyethylene glycol molecule.

Nurko et al investigated the safety of polyethylene glycol in the treatment of chronic constipation in children. They selected children over 2 years and treated them with polyethylene glycol for 8 months. All patients were evaluated for liver and renal functions, electrolytes status, and acid and alkaline states. Its side effects were detected to be $0 \%$ with a confidence coefficient of $95 \%$ (12).

However, constipation is a common problem in pediatrics which can cause negative physical and mental effects on children and their families. In addition, it can be an underlying cause of the other conditions such as infections of the urinary tract, anal and rectal prolapse, child weight reduction, and social and mental problems. The most commonly used standardized treatments include laxatives, but despite their widespread use, the success rate of this therapeutic method is low. Therefore, complementary or alternative therapies should be used to increase this success rate. In recent years, the use of probiotic agents that contain living microorganisms has become widespread, as complementary therapies, for various diseases including digestive disorders. Probiotics are effective in balancing the microbial microflora of the gastrointestinal system. Therefore, this study aimed to comparatively investigate the effect of polyethylene glycol and the simultaneous administration of polyethylene glycol along with probiotics on the treatment of chronic functional constipation in children. The secondary objectives of the study were to comparatively investigate the frequency of stool excretion and painful stool excretion during one week, stool consistency, the frequency of unwanted stool excretion during one week and abdominal pain during one week.

\section{Materials and Methods}

This randomized clinical trial study was conducted on children with chronic functional constipation who referred to Imam Ali Clinic affiliated with Shahrekord University of Medical Sciences during 2017-2018. First, the necessary explanations were presented to the parents regarding the procedure of the study and the informed consent form was signed by the children's legal parents. Both the patient and the nurse were blind to the type of prescribed drugs. However, we assured parents of the children that these drugs were not harmful to them. The individual characteristics of the children were kept confidential. The sample size was determined using the according to the formula below (13):

$$
n=\frac{\left(Z_{1-\frac{\alpha}{2}}+Z_{1-\beta}\right)^{2}\left(S_{1}^{2}+S_{2}^{2}\right)}{d^{2}}=\frac{(1.28+1.96)^{2}(0.49+0.64)}{(0.4)^{2}}=74
$$

The project was approved by the Deputy of Research and Technology of Shahrekord University of Medical Sciences. Then, 150 patients with chronic functional constipation were selected based on the inclusion and exclusion criteria and randomly divided into 2 groups of 75 patients. Randomization was conducted by numbering and coding each patient and 75 patients were randomly selected for the intervention group and 75 patients were selected for the control group (Figure 1). The inclusion criteria were having chronic functional constipation, being 2-15 years of age, and not having large and small bowel disease, and having allergy to polyethylene glycol, bowel obstruction, and kidney and heart diseases. The exclusion criteria included diarrhea and unwanted allergic reactions after the administration of drug. Each group was divided into two subgroups: 2-7 years and 7-15 years old. Additionally, both groups were matched by age, sex, and duration of disease. Both the patient and the nurse were blind to the type of prescribed drugs. However, we assured parents of the children that these drugs were not harmful to them. Group A received $1 \mathrm{~g} / \mathrm{kg}$ of the body's weight of the polyethylene glycol powder with water, and group B received a sachet of probiotic powder (Lactobacillus Rhamnosus, Lactobacillus acidophilus, Lactobacillous Bulgaricus) and $1 \mathrm{~g} / \mathrm{kg}$ of the body weight of water-soluble polyethylene glycol powder. The same dietary recommendations were considered for both groups. In addition, the necessary training on using the toilet was provided. Moreover, children were advised to do physical activities regularly and go to the toilet after having a meal. The ethical considerations of the study were taken into account. Children participated in the study after their mothers provided informed written consent for their participation. The patient information was kept confidential. The necessary information was provided for the patients. The patients who suffered from complications were excluded from the study. Since 


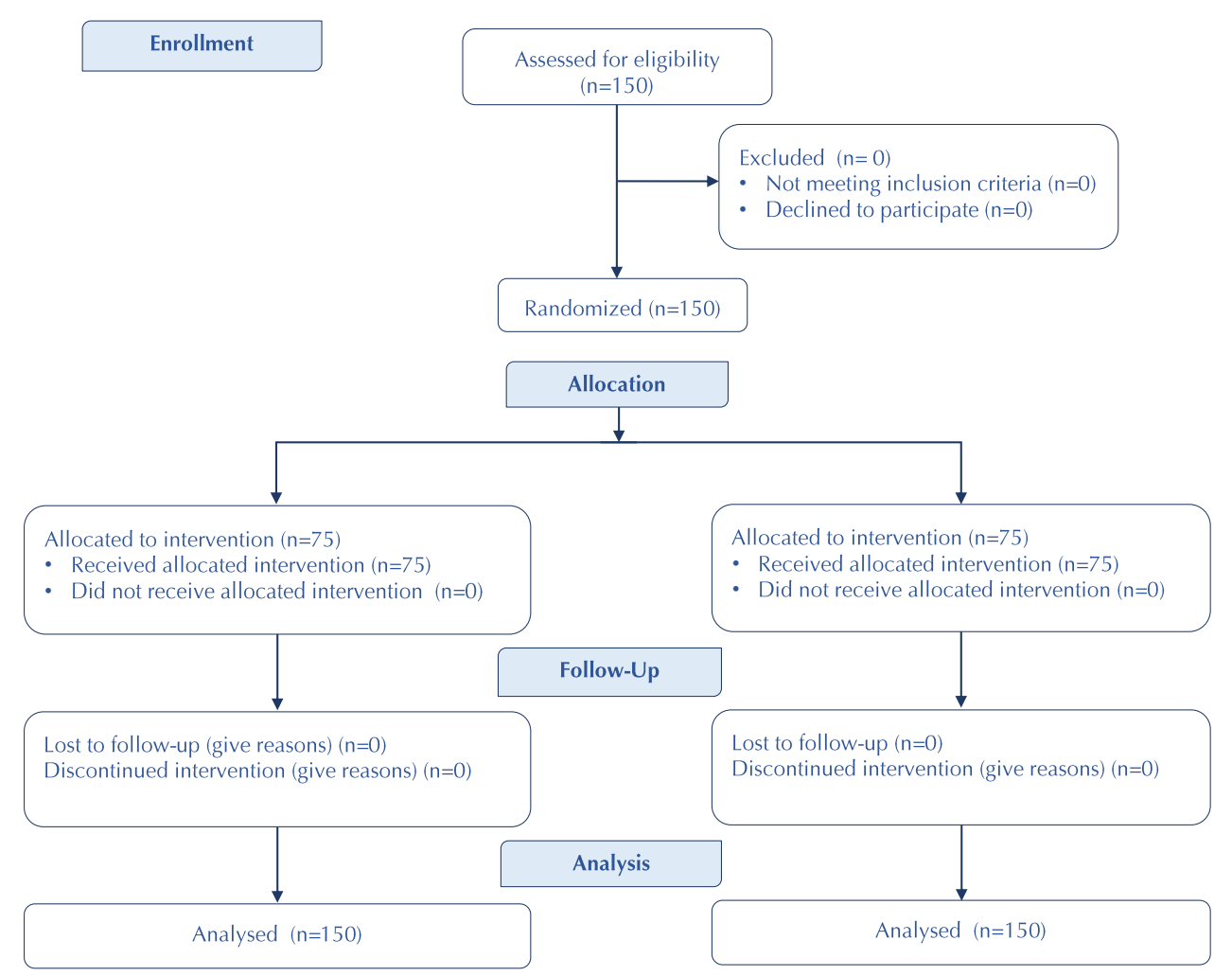

Figure 1. Flow Diagram of the Study Procedure.

there was an intervention in the study, we attempted to avoid any complications in the children. In case of any complication, they were excluded from the study. No charge was imposed on the patients. Questionnaires were completed in weeks 0,1 , and 2 by parents of patients and the data were analyzed by statistical statistics. Data were collected using a checklist. Forms were completed without names. Then, they were encoded and physicians did not see this process. The data was entered into the SPSS. Data were analyzed using descriptive statistics (frequency, percentage, mean, and standard deviation) and analytical statistics (Fisher's exact test). A $P$ value of less than 0.05 was considered statistically significant.

\section{Results}

Each group was divided into two subgroups of 2 to 7 years and 7 to 15 years. Both groups were matched for age, sex, and duration of the disease and there was no significant difference in demographic characteristics between the two groups $(P>0.05)$.

Changes in the frequency of excretion in the first week after the initiation of treatment (week 1) in the first visit did not show a significant difference $(P=0.06)$ (Table 1$)$. Fifty-eight patients $(77 \%)$ in group $\mathrm{A}$ and 54 patients $(72 \%)$ in group B showed changes, indicating that the difference between the two groups based on the Fisher's exact test was not significant $\left(\mathrm{x}_{(2)}^{2}=4.543\right.$ and $\left.P=0.07\right)$. This comparison was done in the second week after treatment using Fisher's exact test, and the differences in the changes in the frequency of excretion between the two groups within one week were not statistically significant $\left(\mathrm{x}_{(2)}^{2}=0.150\right.$ and $\left.P=0.09\right)$ (Table 1$)$.

The changes in stool consistency in the first week after treatment are presented in Table 1. Additionally, the comparison of groups A and B for the changes in the stool consistency during the first week after treatment by Fisher's exact test showed that there was no significant difference $\left(\mathrm{x}_{(1)}^{2}=0.528\right.$ and $P=0.06$ ).

Moreover, this comparison was done in the second week after treatment using Fisher's exact test, and no significant difference in the stool consistency between the two groups was observed $\left(\mathrm{x}_{(2)}^{2}=1.262\right.$ and $\left.P=0.05\right)$.

The data drawn from questionnaires on the frequency of painful excretion per week showed that it increased in the first week of treatment in 56 patients in group A and 55 patients in group B; however, 19 patients in both groups had an unchanged state. This difference between the two groups based on Fisher's exact test was not significant $\left(\mathrm{x}_{(2)}^{2}=1.009\right.$ and $P=0.09$ ) (Table 2 ).

According to the data obtained in the second week of treatment, the frequency of painful excretion in most patients remained unchanged and comparisons did not show any significant difference $\left(\mathrm{x}_{(2)}^{2}=1.171\right.$ and $\left.P=0.07\right)$ (Table 2).

The incidence of fecal incontinence in the first visit did not change in 56 cases and it decreased in 17 cases in group $A$, in 60 patients in group B did not change, and in 14 cases, it reduced. This variable did not change in most of the patients in the second visit and 5 cases in group A 
Table 1. Changes in the frequency of the stool excretion, and the stool consistency (soft or rigid)

\begin{tabular}{|c|c|c|c|c|c|c|}
\hline \multirow{2}{*}{ Variables } & \multirow{2}{*}{ Visit } & \multirow{2}{*}{ Group } & Decrease & Constant & Increase & Sum \\
\hline & & & No. $(\%)$ & No. $(\%)$ & No. $(\%)$ & No. $(\%)$ \\
\hline \multirow{6}{*}{ Stool excretion } & \multirow{3}{*}{ Week 1} & Group B & $0(0)$ & $21(28)$ & $54(72)$ & $75(100)$ \\
\hline & & Group A & $3(4)$ & $14(18.7)$ & $58(77.3)$ & 75 (100) \\
\hline & & Total & $3(2)$ & $35(23.3)$ & $112(74.7)$ & $150(100)$ \\
\hline & \multirow{3}{*}{ Week 2} & Group B & $4(5.3)$ & $67(89.3)$ & $4(5.3)$ & $75(100)$ \\
\hline & & Group A & $4(5.3)$ & $68(90.7)$ & $3(4)$ & $75(100)$ \\
\hline & & Total & $8(5.3)$ & $135(90)$ & $7(4.7)$ & $150(100)$ \\
\hline \multirow{6}{*}{ Stool consistency } & \multirow{3}{*}{ Week 1} & Group B & $70(93.3)$ & $5(6.7)$ & - & $75(100)$ \\
\hline & & Group A & $72(96)$ & $3(4)$ & - & $75(100)$ \\
\hline & & Total & $142(94.7)$ & $8(5.3)$ & - & $150(100)$ \\
\hline & \multirow{3}{*}{ Week 2} & Group B & $3(4)$ & $69(92)$ & - & $75(100)$ \\
\hline & & Group A & $6(8)$ & $65(86.7)$ & - & $75(100)$ \\
\hline & & Total & $9(6)$ & $134(89.3)$ & - & $150(100)$ \\
\hline
\end{tabular}

Table 2. The frequency of painful excretion, abdominal pain and stool incontinence (sudden excretion of gas or liquid)

\begin{tabular}{|c|c|c|c|c|c|c|}
\hline V/ariable & Vicit & Croun & Decrease & Constant & Increase & Sum \\
\hline 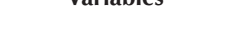 & (1) & (1) & No. $(\%)$ & No. $(\%)$ & No. $(\%)$ & No. $(\%)$ \\
\hline \multirow{6}{*}{ Painful excretion } & \multirow{3}{*}{ Week 1} & Group B & $1(1.3)$ & $19(25.3)$ & $56(73.3)$ & $75(100)$ \\
\hline & & Group A & $0(0)$ & $19(25.3)$ & $56(74.7)$ & $75(100)$ \\
\hline & & Total & $1(7)$ & $38(25.3)$ & $111(74)$ & $150(100)$ \\
\hline & \multirow{3}{*}{ Week 2} & Group B & $1(1.3)$ & $71(94.7)$ & $3(4)$ & $75(100)$ \\
\hline & & Group A & $0(0)$ & $71(94.7)$ & $4(5.3)$ & $75(100)$ \\
\hline & & Total & $1(7)$ & $142(94.7)$ & $7(4.7)$ & $150(100)$ \\
\hline \multirow{6}{*}{ Abdominal pain } & \multirow{3}{*}{ Week 1} & Group B & $4(5.3)$ & $34(45.3)$ & $37(49.3)$ & $75(100)$ \\
\hline & & Group A & $2(2.7)$ & $27(36.0)$ & $46(61.3)$ & $75(100)$ \\
\hline & & Total & $6(4.0)$ & $61(40.7)$ & $83(55.3)$ & $150(100)$ \\
\hline & \multirow{3}{*}{ Week 2} & Group B & $1(1.3)$ & $70(93.3)$ & $4(5.3)$ & $75(100)$ \\
\hline & & Group A & $0(0)$ & $72(96.0)$ & $3(4.0)$ & $75(100)$ \\
\hline & & Total & $1(7)$ & $142(94.7)$ & $7(4.7)$ & $150(100)$ \\
\hline \multirow{6}{*}{$\begin{array}{l}\text { Fecal incontinence } \\
\text { (sudden excretion of } \\
\text { gas or liquid) }\end{array}$} & \multirow{3}{*}{ Week 1} & Group B & $1(1.3)$ & $60(80.0)$ & $14(18.7)$ & $75(100)$ \\
\hline & & Group A & $2(2.7)$ & $56(74.7)$ & $17(22.7)$ & $75(100)$ \\
\hline & & Total & $3(2.0)$ & $116(77.3)$ & $31(20.7)$ & $150(100)$ \\
\hline & \multirow{3}{*}{ Week 2} & Group B & - & $74(98.7)$ & $1(1.3)$ & 75 (100) \\
\hline & & Group A & - & $70(93.3)$ & $5(6.7)$ & $75(100)$ \\
\hline & & Total & - & $144(96.0)$ & $6(4.0)$ & $150(100)$ \\
\hline
\end{tabular}

and 1 case in group B had fecal incontinence (Table 2). The frequency of fecal incontinence also varied between the two groups in the first week $\left(\mathrm{x}_{(2)}^{2}=0.762\right.$ and $\left.P=0.08\right)$ and the second week. There was no significant difference in this variable between the two groups $\left(\mathrm{x}_{(1)}^{2}=2.778\right.$ and $P=0.06)$.

\section{Discussion}

Our study showed that probiotic agents do not have any significant effect on the improvement of the frequency of excretion, bowel incontinence, and abdominal pain, which is inconsistent with the studies conducted on adults; this underlines the hypothesis that constipation in children and adults is significantly different. Our results also showed that there was not any significant difference between groups $\mathrm{A}$ and $\mathrm{B}$ in the variables frequency of stool excretion, stool consistency, painful excretion, abdominal pain, and fecal incontinence. There was no significant relationship between the studied variables and the administration of probiotic agents.

Probiotics contain living microorganisms that appear to be a potential contributor to digestive problems by competing with pathogenic bacteria. The bacteria in these materials, by producing short-chain fatty acids, lactic acid, and acetic acid, reduce the $\mathrm{pH}$ of the colon and change its flora, which in turn affects the movements of the small bowel $(14,15)$. Our study showed that the administration of probiotic agents had no statistically significant effect on the signs of chronic constipation in children. Moreover, in terms of the other clinical outcomes, the differences were generally in favor of probiotics (16). Some of the results of our study were consistent with those of systematic studies on the effects of laxative and diet on the management of chronic constipation in childhood and studies that 
investigated the effects of probiotics (16-19). Therefore, further studies in this regard seem necessary.

Constipation in children differs considerably from that in adults in terms of the incidence, cause, symptoms, treatment, and prognosis $(16,12)$.

In most studies, the administration of probiotics has improved the frequency of excretion, which can be due to the changes in the bowel flora. Studies have also been done to evaluate the intestinal microflora (20). In our study, we combined probiotic materials with polyethylene glycol and did not achieve more promising results. We could not find out whether these two combinations interacted together and neutralized the effect of flora. Our study is in agreement with other studies because each of them (polyethylene glycol and probiotic materials) alone showed positive effects; however, our results regarding their combination in the children are not consistent with other studies $(12,16,20)$. It may be due to participants or flora. Further studies should be done on the amount of flora in the probiotic materials combined with polyethylene glycol and polyethylene alone. Although differences in the improvement of various symptoms can be due to different diets followed by patients, the mixture of probiotics can also explain these differences (21). In addition, the evaluation of patients' condition in some studies was done at longer intervals after treatment and the treatment response was desirable $(11,22)$.

By evaluating the gastrointestinal microflora in patients who consumed probiotic agents and conducting longer and more accurate studies, a new therapeutic method can be designed to control the symptoms of chronic constipation in children. The use of therapeutic strategies that include probiotic drugs may help to achieve more accurate therapeutic guidelines which can be added to current treatments recommended for the management of chronic constipation in children (22).

Our study showed that the administration of probiotic agents combined with polyethylene glycol did not have any significant effect on the improvement of the frequency of excretion, fecal incontinence and abdominal pain compared to polyethylene glycol alone. This difference may be due to the fact that our study was performed on the children and other studies were conducted on the adults $(12,16)$. This point emphasizes that constipation in children is considerably different from that in adults.

\section{Conclusion}

Our results indicate that the administration of probiotic supplement (Lactobacillus Rhamnosus, Lactobacillus acidophilus, Lactobacillous Bulgaricus) has no effect on the improvement of disease in children with chronic constipation. In other words, the comparison of simultaneous administration of the polyethylene glycol with probiotics and polyethylene glycol alone did not show a considerable difference in the treatment of the chronic functional constipation. However, our study had a limitation. The patient's diet was not evaluated due to the lack of adequate time. Hence, further studies are needed to investigate this issue. However, the efficacy of probiotic compounds in improving chronic constipation in children was not considerable.

Conflict of Interests

The authors declare that there is no conflict of interests.

Ethical Approval

This project was approved by the Ethics Committee of Shahrekord University of Medical Sciences with approval number: IR.SKUMS. REC.1397.27.

Authors Contribution

KK prepared the primary draft.MS designed the study. MS completed statistical analyses. SM participated in data collection. kk, MS and SM reviewed and approved the final draft.

\section{Funding/Support}

Deputy of Research and Technology of Shahrekord University of Medical Sciences supported financially the project.

\section{Acknowledgements}

This project was approved by Shahrekord University of Medical Sciences (approval number: 2704). We thank the participants who cooperated with this research project.

\section{References}

1. Hassanzadeh-Keshteli A, Hashemi-Jazi MS, Dehestani D, Adibi P. Epidemiology of constipation and functional constipation and their risk factors in Iranian population. Journal of Isfahan Medical School. 2016;34(404):1282-9. [Persian].

2. Bahardoost $M$, Karimi $S$, Hejrati $A$, Naserifar $F$, Namdar $P$, Chaharmahali A, et al. A comparison between polyethylene glycol and abdominal massage on symptoms and quality of life improvement in functional constipation: a randomize clinical trial study. Journal of Isfahan Medical School. 2018;36(480):557-63. doi: 10.22122/jims.v36i480.10013. [Persian].

3. McCrea GL, Miaskowski C, Stotts NA, Macera L, Varma MG. A review of the literature on gender and age differences in the prevalence and characteristics of constipation in North America. J Pain Symptom Manage. 2009;37(4):737-45. doi: 10.1016/j.jpainsymman.2008.04.016.

4. Mohammad Jafari H, karami H, Rahimikia S, Basiri M. Prevalence of elimination dysfunction, among primary school age children in Sari. Journal of Mazandaran University of Medical Sciences. 2008;18(64):44-51. [Persian].

5. Dupont C, Leluyer B, Maamri N, Morali A, Joye JP, Fiorini JM, et al. Double-blind randomized evaluation of clinical and biological tolerance of polyethylene glycol 4000 versus lactulose in constipated children. J Pediatr Gastroenterol Nutr. 2005;41(5):625-33. doi: 10.1097/01. mpg.0000181188.01887.78.

6. Voskuijl W, de Lorijn F, Verwijs W, Hogeman P, Heijmans J, Mäkel W, et al. PEG 3350 (Transipeg) versus lactulose in the treatment of childhood functional constipation: a double blind, randomised, controlled, multicentre trial. Gut. 2004;53(11):1590-4. doi: 10.1136/gut.2004.043620.

7. Gremse DA, Hixon J, Crutchfield A. Comparison of polyethylene glycol 3350 and lactulose for treatment of chronic constipation in children. Clin Pediatr (Phila). 2002;41(4):225- 


\section{9. doi: $10.1177 / 000992280204100405$.}

8. Zilberberg $\mathrm{MD}$, Shorr $\mathrm{AF}$, Kollef $\mathrm{MH}$. Increase in adult Clostridium difficile-related hospitalizations and casefatality rate, United States, 2000-2005. Emerg Infect Dis. 2008;14(6):929-31. doi: 10.3201/eid1406.071447.

9. Abediny M, Ataiee P, Afkhamzadeh A, Seifmanesh M, Sedaghat B. The effect of probiotics on the treatment of functional constipation in children of 4-12 years of age. Journal of Isfahan Medical School. 2016;33(368):2448-54. [Persian].

10. Nimrouzi $M$, Zarshenas MM. Functional constipation in children: non-pharmacological approach. J Integr Med. 2015;13(2):69-71. doi: 10.1016/s2095-4964(15)60152-2.

11. Alper A, Pashankar DS. Polyethylene glycol: a gamechanger laxative for children. J Pediatr Gastroenterol Nutr. 2013;57(2):134-40. doi: 10.1097/MPG.0b013e318296404a.

12. Nurko S, Youssef NN, Sabri M, Langseder A, McGowan J, Cleveland M, et al. PEG3350 in the treatment of childhood constipation: a multicenter, double-blinded, placebocontrolled trial. J Pediatr. 2008;153(2):254-61, 61.e1. doi: 10.1016/j.jpeds.2008.01.039.

13. Kazem M, Malekafzali H. Statistical Methods and Health Index. Tehran: Moalef Pub; 2017. [Persian].

14. Chmielewska A, Szajewska H. Systematic review of randomised controlled trials: probiotics for functional constipation. World J Gastroenterol. 2010;16(1):69-75. doi: 10.3748/wjg.v16.i1.69.

15. Sadeghzadeh M, Rabieefar A, Khoshnevisasl P, Mousavinasab N, Eftekhari K. The effect of probiotics on childhood constipation: a randomized controlled double blind clinical trial. Int J Pediatr. 2014;2014:937212. doi: $10.1155 / 2014 / 937212$.
16. Tabbers MM, Chmielewska A, Roseboom MG, Crastes $\mathrm{N}$, Perrin C, Reitsma JB, et al. Fermented milk containing Bifidobacterium lactis DN-173 010 in childhood constipation: a randomized, double-blind, controlled trial. Pediatrics. 2011;127(6):e1392-9. doi: 10.1542/peds.2010-2590.

17. Pijpers MA, Tabbers MM, Benninga MA, Berger MY. Currently recommended treatments of childhood constipation are not evidence based: a systematic literature review on the effect of laxative treatment and dietary measures. Arch Dis Child. 2009;94(2):117-31. doi: 10.1136/adc.2007.127233.

18. Bu LN, Chang MH, Ni YH, Chen HL, Cheng CC. Lactobacillus casei rhamnosus Lcr35 in children with chronic constipation. Pediatr Int. 2007;49(4):485-90. doi: 10.1111/j.1442200X.2007.02397.x.

19. Banaszkiewicz A, Szajewska H. Ineffectiveness of Lactobacillus GG as an adjunct to lactulose for the treatment of constipation in children: a double-blind, placebo-controlled randomized trial. J Pediatr. 2005;146(3):364-9. doi: 10.1016/j. jpeds.2004.10.022.

20. Ohland CL, Macnaughton WK. Probiotic bacteria and intestinal epithelial barrier function. Am J Physiol Gastrointest Liver Physiol. 2010;298(6):G807-19. doi: 10.1152/ ajpgi.00243.2009.

21. Gill HS, Guarner F. Probiotics and human health: a clinical perspective. Postgrad Med J. 2004;80(947):516-26. doi: 10.1136/pgmj.2003.008664.

22. Guerra PV, Lima LN, Souza TC, Mazochi V, Penna FJ, Silva AM, et al. Pediatric functional constipation treatment with Bifidobacterium-containing yogurt: a crossover, double-blind, controlled trial. World J Gastroenterol. 2011;17(34):3916-21. doi: 10.3748/wjg.v17.i34.3916. 